\title{
ALTERAÇÃO DE FORMA DENTÁRIA COM RESINA DIRETA: RELATO DE CASO
}

Dental form change with direct resin: case report

Cambio de forma dental con resina directa: Reporte de un caso

Joselúcia da Nóbrega Dias - Doutoranda em Ciências Odontológicas pela Universidade Federal do Rio Grande do Norte-UFRN • Natal-RN • Brasil • E-mail: josynobrega14@gmail.com.

Iasmim Mainny Diógenes Veras - Especialista em Dentística pela Universidade Federal do Rio Grande do Norte-UFRN • Natal-RN • Brasil • E-mail: mainny_iasmim@hotmail.com.

Boniek Castillo Dutra Borges - Professor Assistente do Curso de Graduação em Odontologia e do Programa de Pós Graduação em Ciências Odontológicas da Universidade Federal do Rio Grande do Norte-UFRN • Natal-RN • Brasil • E-mail: boniek.castillo@gmail.com.

Lilian Karine Cardoso Guimarães de Carvalho • Doutora em Ciências Odontológicas pela Universidade Federal do Rio Grande do Norte-UFRN • Natal-RN • Brasil • Email: liliankarinec@yahoo.com.br.

Isauremi Vieira de Assunção • Professora Assistente do Curso de Graduação em Odontologia, Especialização em Dentística e do Programa de Pós Graduação em Ciências Odontológicas da Universidade Federal do Rio Grande do Norte-UFRN • Natal-RN• Brasil • E-mail: isauremi@gmail.com.

Autora responsável pela correspondência:

Joselúcia da Nóbrega Dias • E-mail: josynobrega14@gmail.com 


\section{ciênciála
pural}

\section{RESUMO}

Introdução: A melhoria da qualidade dos materiais restauradores odontológicos, sobretudo das resinas compostas, permitiu a resolução de diversos problemas estéticos de forma conservadora, rápida e satisfatória. Objetivo: Este trabalho objetivou relatar um caso clínico de alteração de forma dos dentes anteriores, solucionado com resina composta pela técnica direta. Descrição do Caso: A paciente apresentava incisivos laterais ovalados e restauração classe IV insatisfatória no incisivo central superior direito. Após exame clínico e seleção de cor, os dentes 12, 12, 21 e 22 foram reanatomizados com resina composta direta através da técnica estratificada, além da substituição da restauração classe IV. Para uma perfeita mimetização dos elementos dentários, foram utilizadas resinas translúcidas, opacas e de efeito opalescente, além de resinas para reproduzir as camadas de esmalte e dentina. Conclusões: Uma boa seleção do material e aplicação correta da técnica, permitem restaurações imperceptíveis, com excelente nível de texturização, acabamento e polimento, devolvendo a harmonia do sorriso.

Palavras-Chave: Estética Dentária. Restauração Dentária Permanente. Materiais Dentários.

\section{ABSTRACT}

Introduction: The improvement of the quality of dental restorative materials, especially of composite resins, allowed the resolution of several aesthetic problems in a conservative, fast and satisfactory way. Objective: This study aimed to report a clinical case of alteration of the shape of the anterior teeth, solved with resin composed by the direct technique. Case Description: The patient had oval lateral incisors and an unsatisfactory class IV restoration on the upper right central incisor. After clinical examination and color selection, teeth 12, 12, 21 and 22 were reanatomized with direct composite resin using the stratified technique, in addition to replacing the class IV restoration. For perfect mimicry of dental elements, translucent, opaque and opalescent resins were used, in addition to resins to reproduce the enamel and dentin layers. Conclusions: A good selection of the material and correct application of the technique, allow imperceptible restorations, with an excellent level of texturing, finishing and polishing, returning the harmony of the smile.

Keywords: Dental Esthetics. Permanent Dental Restoration. Dental Materials.

\section{ESUMEN}

troducción: La mejora de lacalidad de losmateriales de restauración dental, pecialmente de las resinas compuestas, permitió la resolución de varios problemas éticos de forma conservadora, rápida y satisfactoria. Objetivo: Este estudio tuvo no objetivo informar un caso clínico de alteración de la forma de los dientes teriores, resuelto con resina compuesta por la técnica directa. Descripción del caso: 
El paciente tenía incisivos laterales ovales y una restauración de clase IV insatisfactoria en el incisivo central superior derecho. Después del examen clínico y la selección del color, los dientes 12, 12, 21 y 22 se reanatomizaron con resina compuesta directa utilizando la técnica estratificada, además de reemplazar la restauración de clase IV. Para una perfecta imitación de los elementos dentales, se utilizaron resinas translúcidas, opacas y opalescentes, además de resinas para reproducir las capas de esmalte y dentina. Conclusiones: Una buena selección del material y la correcta aplicación de la técnica, permiten restauraciones imperceptibles, con un excelente nivel de texturizado, acabado y pulido, devolviendo la armonía de la sonrisa.

Palabras clave: Estética Dental. Restauración Dental Permanente. Materiales Dentales. 


\section{Introdução}

Alterações de forma, cor e posicionamento por problemas anatômicos representam um dos principais motivos para a insatisfação com o sorriso. Atualmente, as resinas compostas representam o material de primeira escolha para restaurar dentes anteriores, principalmente devido às suas propriedades estéticas, preservação de estrutura dentária sadia e bom desempenho clínico a longo prazo ${ }^{1,2}$.

Além disso, as resinas compostas são excelentes materiais para restaurações diretas, pois permitem a execução de um procedimento conservador, previsível, reparável e de baixo custo. Ao longo dos anos, esse material passou por evoluções em suas características químicas e físicas, o que permitiu às resinas atuais melhores propriedades ópticas e mimetização precisa da translucidez e opacidade do elemento dentário ${ }^{3}$.

As técnicas restauradoras adesivas possibilitam procedimentos aditivos ou minimamente invasivos e estão sendo cada vez mais utilizadas, uma vez que é compreendido que a adesão sobre o esmalte é maior quando comparada a dentina, propiciando técnicas com menor ou nenhum desgaste dentário. Logo, a preservação da estrutura do esmalte é fundamental para a longevidade da restauração ${ }^{4}$.

Essa evolução dos materiais restauradores foi atrelada ao desenvolvimento de novas técnicas para atender à exigência estética dos pacientes, ampliando as opções de tratamento. Dentre essas técnicas atuais destaca-se a estratificação em resina composta, que pode ser simples ou avançada (estratificação tridimensional), sendo sua execução dependente da análise minuciosa das características dentárias que serão reproduzidas na restauração ${ }^{5}$. As técnicas de estratificação permitem alterações de cor nos tecidos dentários associadas à fluorescência, opalescência e contra-opalescência e permitem criar efeitos cromáticos que têm como vantagem a mimetização e reprodução dos elementos dentários através das resinas compostas ${ }^{6}$.

Dessa forma, a técnica de estratificação associa tecnologia com arte, através da nimetização do policromatismo dentário de forma não invasiva e reversível, com evisibilidade de resultado, menor custo e maior facilidade de manipulação7. 
Este trabalho objetivou apresentar um caso de alteração de forma dentária com resina composta, que muito incomodava a paciente, e o sucesso alcançado com a resina composta realizada de forma imperceptível.

\section{Relato de Caso}

Paciente do sexo feminino, 23 anos, procurou o serviço de atendimento do Curso de Especialização em Dentística da Universidade Federal do Rio Grande do Norte queixando-se da estética do seu sorriso. Após anamnese, exame clínico e protocolo fotográfico se observou que os incisivos laterais possuíam formato ovalado e o incisivo central superior direito apresentava restauração classe IV insatisfatória (figura 1). Com isso, foram planejadas reanatomizações estéticas com resina composta direta nos elementos dentários 12, 11, 21 e 22, além da substituição da restauração classe IV.

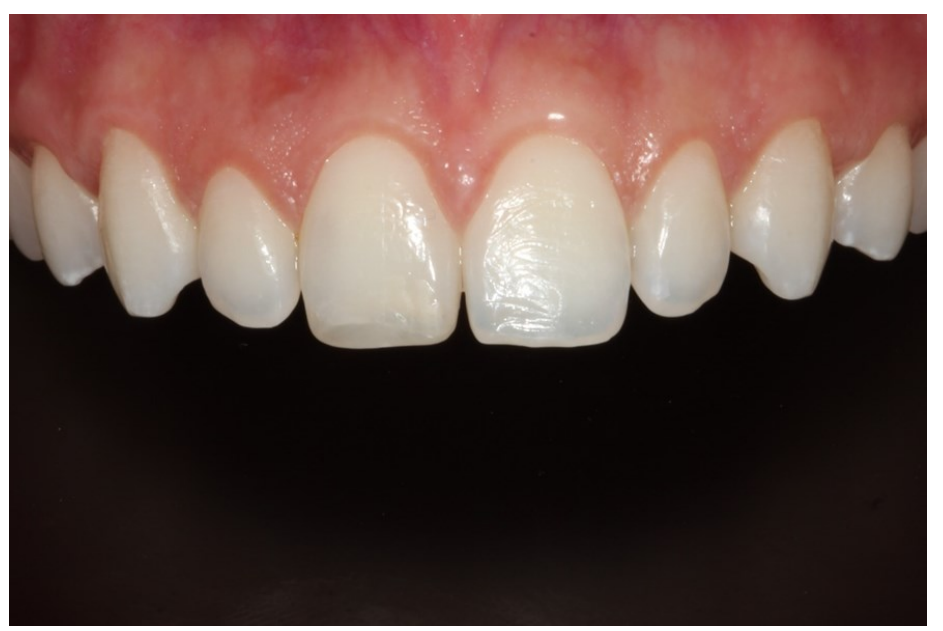

Figura 1. Aspecto inicial: Formato ovalado dos dentes anterossuperiores e classe IV insatisfatória no elemento 11. Natal-RN, 2020.

Após profilaxia com pedra-pomes e água, foi realizada a seleção de cor por meio

da Escala Vita e, logo em seguida, os elementos dentários foram isolados utilizando o damento semiabsoluto. $\mathrm{O}$ tratamento restaurador foi iniciado com $\mathrm{o}$ dicionamento com ácido fosfórico (Condac 37\%, FGM, SC, Brasil) por 15 segundos 
seguido de lavagem e secagem (figura 2). Em seguida, foi aplicado o sistema adesivo (Ambar APS, FGM, SC, Brasil) e fotoativado por 20 segundos (figura 3).

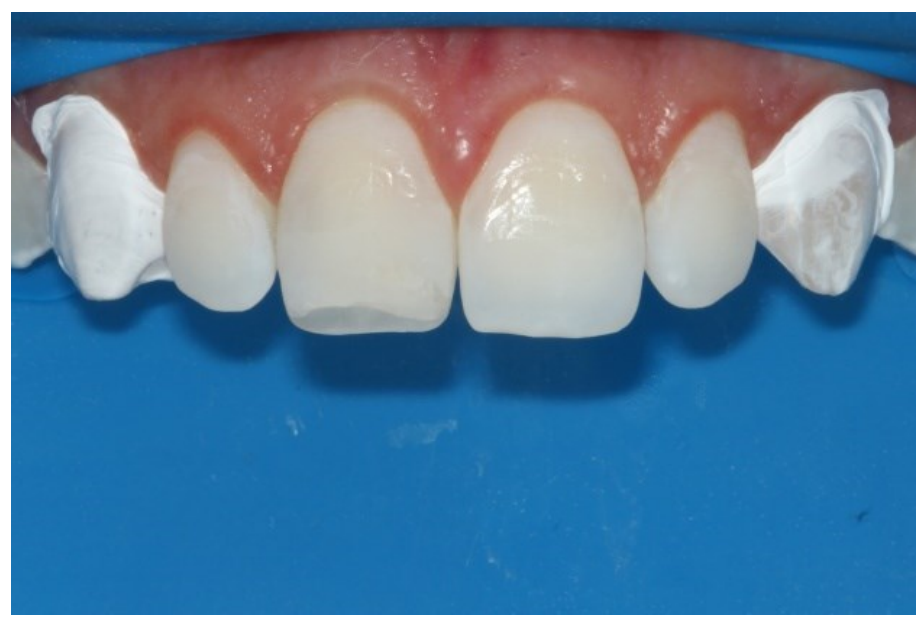

Figura 2. Aspecto após condicionamento ácido. Natal-RN, 2020.

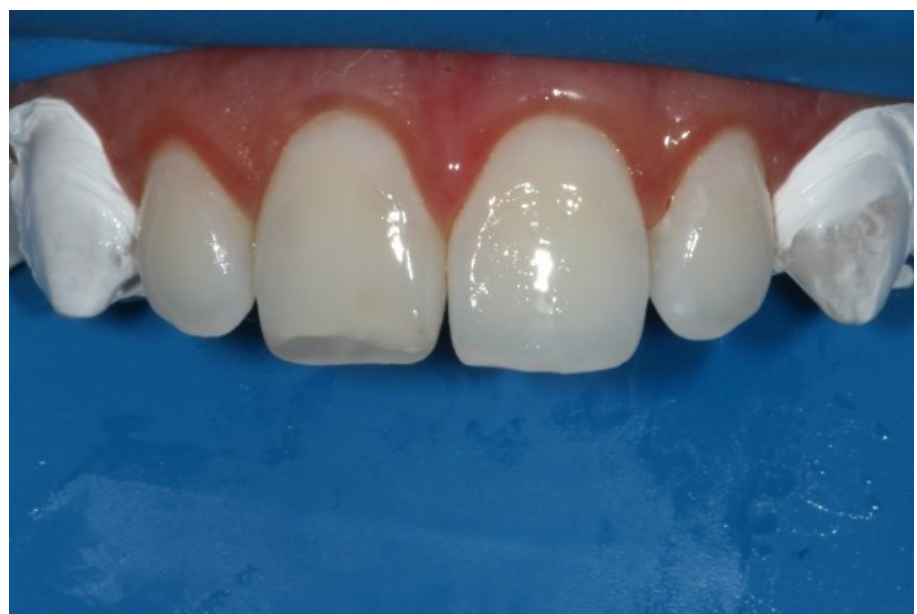

Figura 3. Aspecto após aplicação do sistema adesivo. Natal-RN, 2020.

A restauração foi realizada pela técnica de inserção incremental, na qual cada incremento de $2 \mathrm{~mm}$ de resina composta foi polimerizado por 20 segundos com o aparelho de LED Valo (Ultradent, South Jordan, Utah, USA). Para a reanatomização dos elementos dentários, a técnica de estratificação foi iniciada pela reprodução do esmalte palatino e da camada de esmalte da borda incisal com resina translúcida TRANS N (Vittra APS, FGM, SC, Brasil). Em seguida, foi realizada a confecção do halo incisal opaco com a resina na cor DAO (Vittra APS, FGM, SC, Brasil). A camada referente à dentina para a caracterização dos mamelos dentinários foi confeccionada com resina na cor DA1 (Vittra APS, FGM, SC, Brasil) e a região que fica entre os mamelos e o halo opaco foi construída com resina de efeito opalescente na cor TRANS 


\section{ciênciála
pural}

OPL (Vittra APS, FGM, SC, Brasil). A última camada, referente ao esmalte, foi confeccionada com a resina na cor E-Bleach (Vittra APS, FGM, SC, Brasil). Realizou-se ainda o acabamento e polimento com borrachas abrasivas e discos de feltro (Kit Diamond Master, FGM, SC, Brasil) (figura 4). As restaurações obtiveram excelente resultado estético na modificação da forma dos elementos dentários e a paciente ficou bastante satisfeita com o aspecto final.

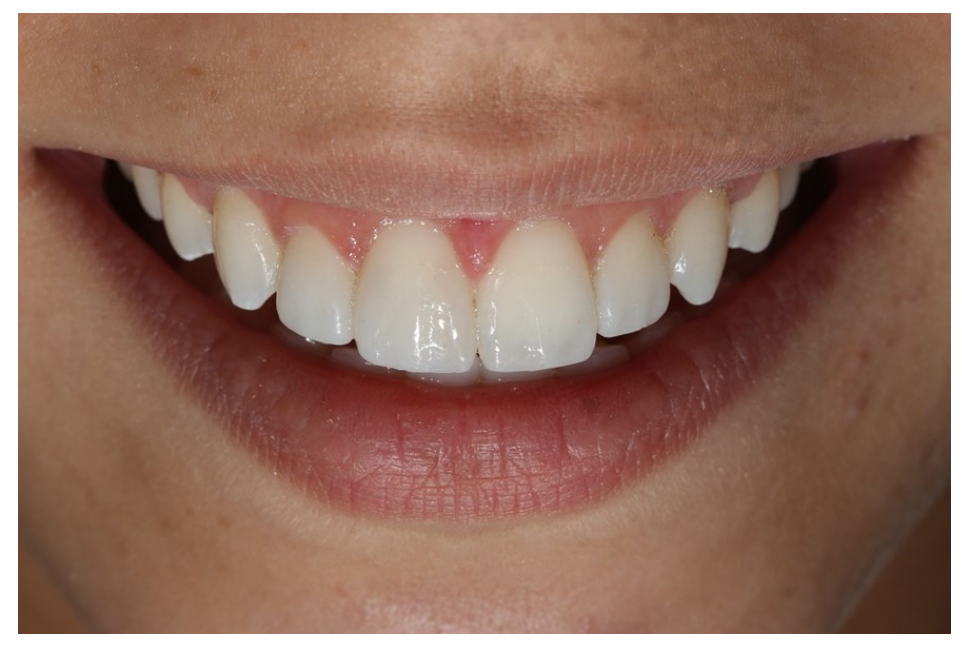

Figura 4. Aspecto final após reanatomização com resina composta e substituição da classe IV insatisfatória. Natal-RN, 2020.

\section{Discussão}

A grande aplicabilidade clínica dos compósitos é possível devido a condições clínicas favoráveis de desempenho (longevidade e manutenção da estética), maior preservação de tecidos dentários e formação de um corpo único, mimetizando a estrutura dentária. Com a evolução dos materiais restauradores, as resinas compostas passaram a ser amplamente utilizadas tanto em restaurações anteriores como posteriores por apresentarem adequada resistência mecânica, alta resistência ao desgaste e retenção do polimento por maior período de tempo ${ }^{8}$.

Nesse caso de mudança de forma, optou-se pela técnica direta em resina omposta por tratar-se de uma paciente jovem e pela possibilidade de realização da enica aditiva sem nenhum desagaste, já que a adesão foi realizada em esmalte. Os tes naturais apresentam variações anatômicas e diferentes texturas superficiais que 


\section{ciênncia
piural}

podem influenciar a seleção de cor. Diante disso, o efeito da técnica de estratificação de esmalte sobre dentina e os diferentes graus de translucidez dos dentes naturais tornam-se desafios para a reanatomização de dentes anteriores ${ }^{9}$.

$\mathrm{O}$ advento da nanotecnologia permitiu às resinas compostas atuais uma maior estabilidade de cor e características ópticas que facilitam a técnica de escultura e permitem excelente polimento e maior resistência ao desgaste, garantindo longevidade do tratamento ${ }^{10,11}$. Portanto, é importante destacar que, além do tamanho de suas partículas, os compósitos de resina também são classificados de acordo com o grau de translucidez, indicação e localização na restauração, o que facilita a escolha do material para melhor reprodução dos tecidos dentários ${ }^{6}$.

Além disso, em casos de reanatomização e restabelecimento de ponto de contato deve-se levar em consideração a expectativa do paciente e conhecimento do profissional para escolha da melhor técnica para obter um resultado satisfatório ${ }^{12}$. Através da utilização da estratificação no uso das resinas, é possível conseguir reproduzir o efeito óptico natural do elemento dentário, permitindo uma mudança de forma dentária sem a percepção da presença do material restaurador.

\section{Conclusão}

Através da técnica de estratificação de resina composta, foi possível solucionar a queixa relatada pela paciente através da reanatomização dos seus elementos dentários anterossuperiores de forma eficiente, devolvendo estética e função.

\section{Referências}

1. Demarco FF, Collares K, Coelho-de-Souza FH, Correa MB, Cenci MS, Moraes RR, Opdam NJM. Review Anterior composite restorations: A systematic review on long-term survival and reasons for failure. Dental Materials. 2015;31(10):1214-1224. 
2. Demarco FF, Collares K, Corra MB, Cenci MS, Moraes RR, Opdam NJM. Should my composite restorations last forever? Why are they failing? Braz. Oral Res. 2017;31(56):92-99.

3. Romero MF. Esthetic anterior composite resin restorations using a single shade: Step-by-step technique. The Journal of Prosthetic Dentistry. 2015;114(1):9-12.

4. Calichio L, Kyrillos M, Moreira M, Giordani G, Cabral F,Souza A. Minimally invasive dentistry applied to esthetic transformation of the smile. J Clin Dent Res. 2016;13(4):53-64.

5. Reis A, Higashi C, Loguercio AD. Re-anatomization of anterior eroded teeth by stratification with direct composite resin. J Esthet Restor Dent. 2009;21(5):30416.

6. Baratieri LN, Araújo E, Monteiro JR S. Color in Natural Teeth and Direct Resin Composite Restorations: Essential Aspects. The European Journal of Esthetic Dentistry. 2007;2(2):172-86.

7. Pontons-Melo JC, Furuse AY, Mondelli J. A direct composite resin stratification technique for restoration of the smile. Quintessence Int. 2011; 42(3):205-11.

8. Ferracane JL. Resin composite--state of the art. Dental Materials. 2011;27(1):2938.

9. Bayindir F, Gozalo-Diaz D, Kim-Pusateri S, Wee AG. Incisal Translucency of Vital Natural Unrestored Teeth: A Clinical Study. Journal of Esthetic and Restorative Dentistry. 2012; 24 (5): 335-43.

10. Pereira DA, Borges MG, Silva FP, Menezes MS. Esthetic smile rehabilitation throught direct restorative procedure with nanoparticulate composite resin: a case report. Rev Odontol Bras Central. 2016; 25(72): 54-58.

11. Alzraikat H, Burrow MF, Maghaireh GA, Taha NA. Nanofilled Resin Composite Properties and Clinical Performance: A Review. Oper Dent. 2018;43(4):173-190.

12. Bandéca MC, Calixto LR, Tonetto MR, Pinto SCS, Saad JRC. Fechamento de diastema com resina composta. Revista Clínica - International Journal of Brazilian Dentistry. 2011;7(2):156-164. 